\title{
Supercontinuum white light lasers: a review on technology and applications
}

\section{Nicolai Granzow}

Nicolai Granzow, "Supercontinuum white light lasers: a review on technology and applications," Proc. SPIE 11144, Photonics and Education in Measurement Science 2019, 1114408 (17 September 2019); doi: $10.1117 / 12.2533094$

SPIE Event: Joint TC1 - TC2 International Symposium on Photonics and Education in Measurement Science 2019, 2019, Jena, Germany 


\title{
Supercontinuum white light lasers: a review on technology and applications
}

\author{
Nicolai Granzow ${ }^{\mathrm{a}}$ \\ ${ }^{a}$ NKT Photonics, Schanzenstr. 39 Bldg. D9-D13, 51063 Cologne, Germany
}

\begin{abstract}
Supercontinuum "White Light Lasers" have become a well-established turn-key fiber-laser technology addressing a wide range of applications from bio-medical-imaging to optical device characterization, where they add value due to their unique combination of optical parameters, including an extremely wide spectral coverage from $400 \mathrm{~nm}$ to $2400 \mathrm{~nm}$, several W of optical output power, and a perfect Gaussian beam that can be focused down to the diffraction limit. This review introduces the state-of-the-art technology and some applications.
\end{abstract}

Keywords: Lasers, Supercontinuum, White Light, Light Source, Broadband, Imaging, Optical Metrology, NanoPhotonics

\section{INTRODUCTION}

Supercontinuum lasers are a technology that combines some of the properties of conventional broadband light sources (like Halogen lamps, Xenon lamps, laser-driven light sources, superluminescent light emitting diodes etc.) with the unique properties of single-mode lasers: an extremely broad spectral coverage from $\sim 400 \mathrm{~nm}$ to $2400 \mathrm{~nm}$ emitted as a continuum with an integrated power of up to several W (even tens of W), and at the same time a perfect Gaussian singlemode beam which can be focused down to the diffraction limit, giving rise to a brightness that can exceed conventional light sources by more than six orders of magnitude, while staying within the spatial resolution regime of optical microscopy if needed.

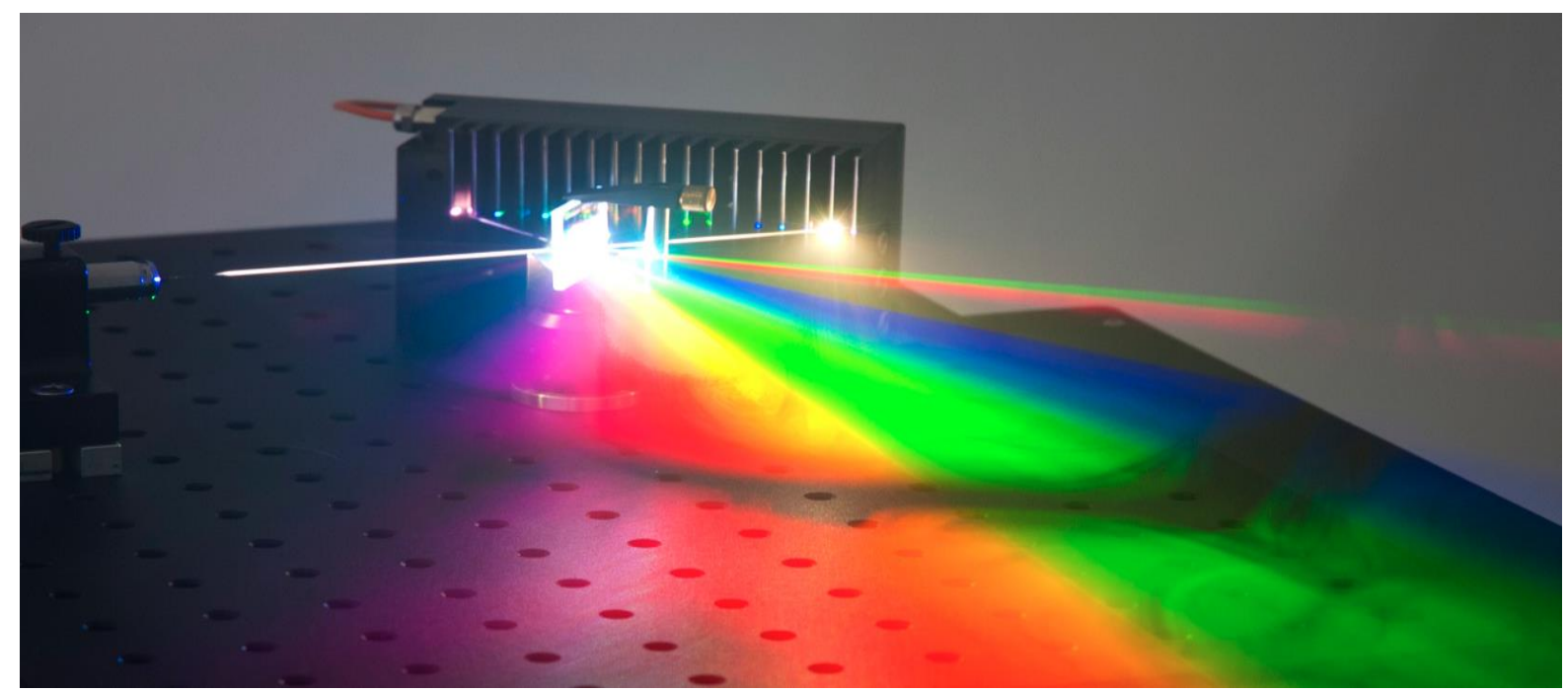

Figure 1. Output of a supercontinuum laser dispersed by a prism in order to visualizing the continuous spectrum in the visible part of the spectrum.

This unique combination of optical properties can add value within many applications related to modern science, and as of today there is no other technology that provides high power and extremely broadband (or widely tunable) light with ps pulse duration, emitted from a single-mode fiber. 
Supercontinuum laser have left their niche within nonlinear fiber optics more than 15 years ago and evolved to become a mature laser technology with turn-key operation and several thousand systems spread across scientific labs worldwide. Besides well-known applications within bio and medical imaging (for example fluorescence confocal microscopy, fluorescence lifetime imaging microscope, super-resolution microscopy, optical coherence tomography or flow cytometry), more and more new applications areas have come up over the past decade, including steady-state or timeresolved measurement techniques within plasmonics, metamaterials, $2 \mathrm{~d}$ layered materials, carbon nanotubes, quantum dots, nano-optics, physical chemistry or spectroscopy, along with a wide range of applications within industrial metrology and inline process monitoring.

\section{SUPERCONTINUUM GENERATION}

Supercontinuum generation ${ }^{1}$ is the formation of broad continuous spectra by propagation of high-power pulses through nonlinear media and was first observed in 1970 by Alfano and Shapiro ${ }^{2,3}$.

The term supercontinuum does not refer to a specific phenomenon but rather to a plethora of nonlinear effects which, in combination, lead to considerable spectral broadening of optical pulses and thereby potentially octave-spanning output. The involved nonlinear effects depend on the dispersion in the material and count effects like self-phase modulation (SPM), Raman scattering, phase matching and solitons dynamics.

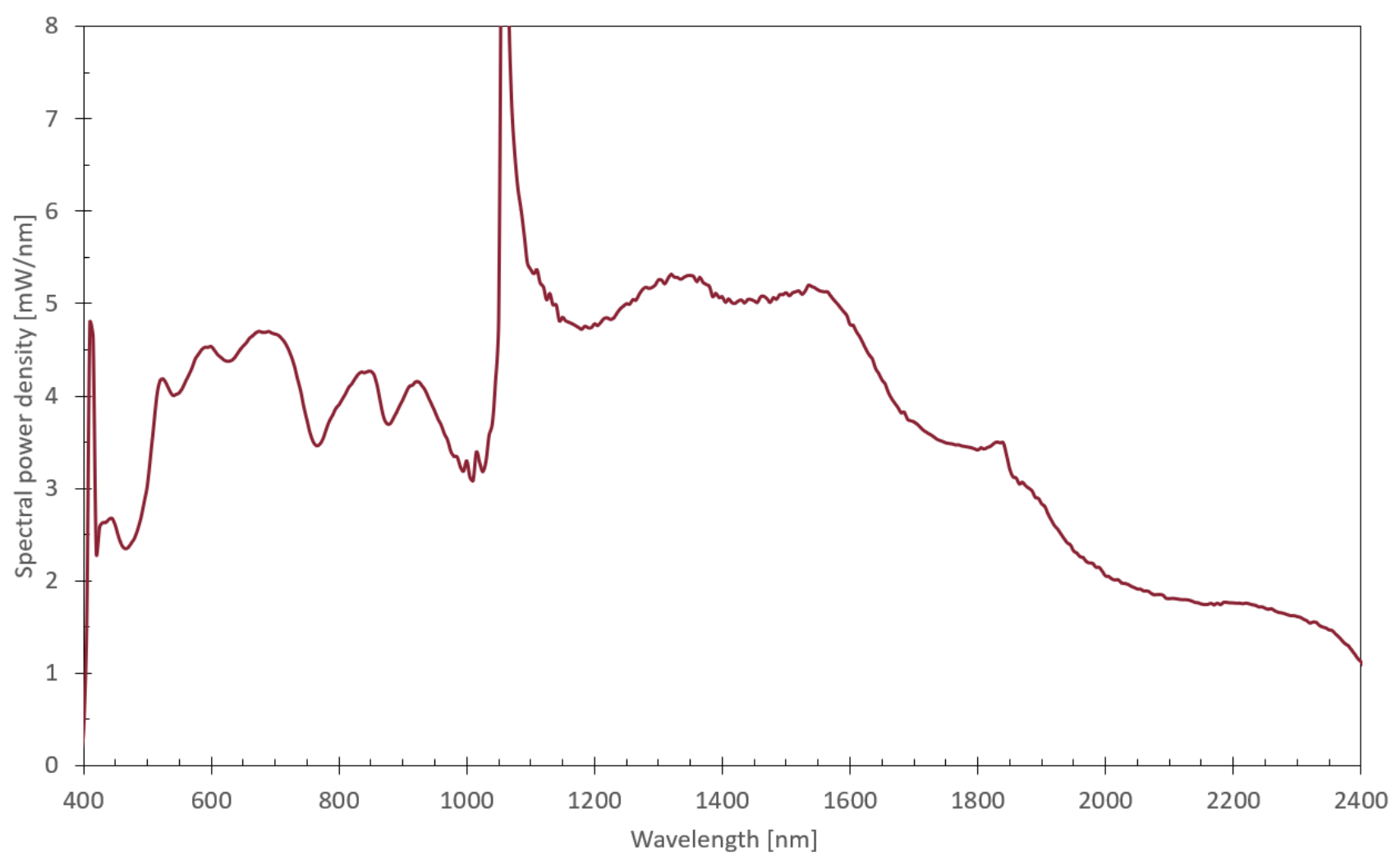

Figure 2. Spectral power density of a commercial supercontinuum laser (SuperK FIANIUM FIU-15, NKT Photonics), covering wavelengths from $400 \mathrm{~nm}$ to $2400 \mathrm{~nm}$ with around $6 \mathrm{~W}$ integrated power. The $1064 \mathrm{~nm}$ spike is the residual pump peak of the $\mathrm{Yb}$ fiber laser.

Provided enough power is available, supercontinuum generation can be observed in a drop of water or even air, but the nonlinear effects involved in the spectral broadening are highly dependent on the dispersion of the media and clever 
dispersion design can significantly reduce power requirements. The widest spectra are obtained when the pump pulses are launched close to the zero-dispersion wavelength of the nonlinear media, and the introduction of the nonlinear photonic crystal fibers (details in section 3) with zero-dispersion wavelengths in range of the most commonly available pump laser wavelengths (Ti:Sa lasers or Yb fiber lasers for instance) led to a boom in supercontinuum experiments.

Supercontinuum light can be best described as 'broad as a lamp, bright as a laser' (Fig. 2). Incandescent and fluorescent lamps, such as those made from tungsten halogens or xenon, provide a very broad spectrum, typically $400 \mathrm{~nm}$ to $1700 \mathrm{~nm}$, but the intensity is limited to the quality of the filament or the efficiency of the gas excitation. Furthermore, as the light is not spatially coherent, coupling the light into the fiber is a challenging affair, resulting in a low-power, lowbrightness source with mediocre beam quality. Lasers on the other hand have high spatial coherence and very high brightness, which enables optimum coupling to a fiber and outstanding single-mode beam quality. However, lasers are usually monochromatic, and thus if more than one wavelength is needed extra lasers at specific wavelengths are required to cover a broad spectrum. A supercontinuum source bridges this gap, providing an ultrabroadband white-light spectrum but with single-mode beam characteristics and excellent pointing stability and the brightness of a laser. The figure below shows the broad emission spectrum that can be achieved.

\section{NONLINEAR PHOTONIC CRYSTAL FIBERS}

Efficient supercontinuum generation relies on a nonlinear medium with tailored dispersion and nonlinearity - essentially all commercial supercontinuum lasers consist of a pulsed pump laser and a specially designed microstructured fiber acting as the nonlinear medium: a highly nonlinear photonic crystal fiber (PCF).

The term photonic crystal fiber is inspired by the unique cladding structure of this fiber class. Standard fibers guide light by total internal reflection between a core with a high refractive index (typically germanium doped silica), embedded in a cladding with a lower index (typical pure or fluorine-doped silica). The index differences in PCFs are obtained by forming a matrix of different materials with high and low refractive index. In this way, a hybrid material is created with properties not obtainable in solid materials (e.g. very low index or novel dispersion). The hybrid material cladding can be constructed with a structure similar to the one found in certain crystals, which is where the term photonic crystal fiber originates. The fibers are not fabricated in crystalline materials as the name might indicate, most commonly they consist of high purity fused silica and air holes. There are two fundamental classes of PCFs: index-guiding PCFs and fibers that confine light through a photonic bandgap - supercontinuum generation relies on index-guiding fibers.

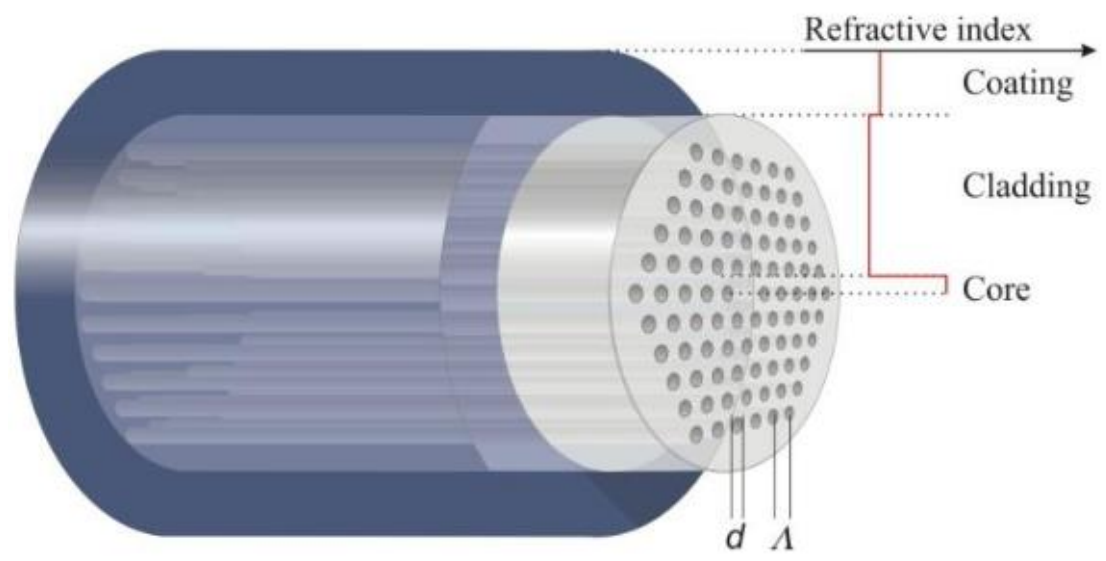

Figure 3. Schematic of the classical triangular cladding single-core photonic crystal fiber in which light is guided in a solid core embedded in a triangular lattice of air holes. The fiber structure is determined by the hole-size, d, and the hole-pitch, $\Lambda$. Like standard fibers, the PCF is coated with a high index polymer for protection and to strip off cladding-modes. 
An index guiding PCF comprises a solid glass high-index core embedded in an air-filled cladding structure where a number of air holes are arranged in a pattern that runs along the length of the fiber, creating a hybrid air-silica material with a refractive index lower than the core. This air-silica matrix structure has given rise to several other names like microstructured and holey fibers, but despite the difference in terminology, they all refer to the same fiber type.

Figure 3 shows the basic principle of the classical triangular cladding single-core photonic crystal fiber, which has proven to be one of the most efficient and flexible designs, forming the basis of most PCFs today. The outer diameter of the fibers is typically $125 \mu \mathrm{m}$ and the pitch of the fiber in the figure is consequently $10-15 \mu \mathrm{m}$.

Nonlinear fibers are typically designed with a pitch of approximately 1-3 $\mu \mathrm{m}$, and therefore, the microstructured region of a nonlinear PCF only takes up the inner $20-50 \%$ of the fiber cross section. Compared to the well-known standard fiber, one of the most novel features of the triangular PCFs is the possibility to design fibers which exhibit no secondorder mode cut-off, rendering them single-mode at any wavelength. This fiber type is known as endlessly single-mode fibers and can be realized by choosing sufficiently small holes in the cladding ${ }^{4,5}-$ in fact this is the reason why PCFbased supercontinuum lasers are capable of providing a $2000 \mathrm{~nm}$ wide spectrum with a Gaussian beam profile at any wavelength.

\section{COMMERCIAL SUPERCONTINUUM LASERS AND APPLICATIONS}

The majority of commercial supercontinuum lasers are fully fiber-based systems consisting of a modelocked fiber oscillator as the master seed laser, providing ps pulses at $\sim 1064 \mathrm{~nm}$ and repetition rates in the tens of $\mathrm{MHz}$ regime, injected into a fiber amplifier giving rise to high peak power, and finally a few meters of a specially designed indexguiding PCF with suitable dispersion landscape. The resulting spectra have a cut-in wavelength around $400 \mathrm{~nm}$, and extent into the infrared up to $\sim 2400 \mathrm{~nm}$ (where the transmission window of the fused-silica PCF begins to close off), with several $\mathrm{W}$ of integrated power and a Gaussian beam profile with $\mathrm{M}^{2}<1.1$ at any wavelength.

When combined with a tunable spectral filter, the supercontinuum source is transformed into a widely tunable laser, making it a versatile laser tool for a wide range of applications. Besides conventional grating-based monochromators used for filtering, tunable filters are available that rely on acousto-optical tunable filters (AOTFs), linear gradient filters or holographic filters - each filter technology providing specific characteristics regarding tuning range, linewidth, out-ofband-suppression, tuning rate or the number of colors accessible simultaneously - such that ultimately the combination of the supercontinuum laser and tunable filter determine the specifications of the full system.

One of the main drivers of supercontinuum technology was its use for bio and medical imaging (emerging from 2003 on), where supercontinuum lasers allow illuminating, interrogating or exciting biological materials with a diffraction limited laser beam with literally no limitation on the choice of wavelength(s). Besides confocal microscopy (Leica Microsystems TCS SP8 X based on a supercontinuum laser ${ }^{6}$ ) a wide range of techniques have become established today, including fluorescence lifetime imaging microscopy (FLIM), Förster resonance energy transfer (FRET), optical coherence tomography (OCT), scanning laser ophthalmology (SLO), flow cytometry or super-resolution techniques such as stimulated emission depletion microscopy (STED).

Wavelength tunability and a diffraction limited beam are also important requirements within optical characterization of nano-scale structures, for instance in plasmonics, nano-structured materials, quantum dots, vacancy centers, 2dimensional layered media (such as Graphene, $\mathrm{MoS}_{2}$ etc.), carbon nanotubes or semiconductor nano devices, where supercontinuum lasers have become an optical tool for reflection / absorption measurements, photo-current measurements, photoluminescence, time-resolved techniques (time-correlated single-photon counting, time-of-flight,...), interferometric techniques (such as optical coherence tomography for non-destructive inspection) or combined with scanning microscopy methods (scanning optical near-field microscopy, atomic force microscopy, scanning tunneling microscopy,...).

Finally, supercontinuum lasers can replace conventional light sources or single-line lasers within (mostly industrial) inspection, non-destructive testing, component characterization or sensor calibration, where they add value due to their combination of wavelength tunability, brightness and spatial beam profile. 


\section{REFERENCES}

[1] NKT Photonics "Supercontinuum Generation" https://www.nktphotonics.com/lasers-fibers/technology/supercontinuum/ (25.04.2019)

[2] R. R. Alfano and S.L. Shapiro, "Emission in the region 4000 to $7000 \AA$ via four-photon coupling in glass" Physical Review Letters 24, 584 (1970).

[3] R. R. Alfano and S.L. Shapiro, "Observation of self-phase modulation and small-scale filaments in crystals and glasses" Physical Review Letters 24, 592 (1970).

[4] T. A. Birks, J. C. Knight and P. St.J. Russell, "Endlessly single-mode photonic crystal fiber" Opt. Letters 22, 961 (1997).

[5] B. T. Kuhlmey, "Microstructured optical fibers: where's the edge?" Opt. Express 10, 1285 (2002)

[6] Leica Microsystems https://www.leica-microsystems.com/de/produkte/konfokalmikroskope/p/leica-tcs-sp8-x/ $(25.04 .2019)$ 